\title{
Stereo Video Transmission Using LDPC Code
}

\author{
Rui GUO, Lixin WANG, Xiaoxia JIANG \\ Communication College, Hangzhou Dianzi University, Hangzhou, China \\ Email: hbsyrgr@yahoo.com.cn \\ Received on April 16, 2008; revised and accepted on August 18, 2008
}

\begin{abstract}
Stereo video is widely used because it can provide depth information. However, it is difficult to store and transmit stereo video due to the huge data amount. So, high efficient channel encoding algorithm and proper transmission strategy is needed to deal with the video transmission over limited bandwidth channel. In this paper, unequal error protection (UEP) based on low density parity check (LDPC) code was used to transmit stereo video over wireless channel with limited bandwidth. Different correction level LDPC code was used according to the importance of video stream to reconstruction at the receiver. Simulation result shows that the proposed transmission scheme increases the PSNR of reconstructed image, and improves the subjective effect.
\end{abstract}

Keywords: Stereo Video, LDPC Code, UEP, Data Partion, EEP

\section{Introduction}

Stereo video is widely used because it can provide depth information in stereoscopic television, video conference, remote control, telemedicine and other fields $[1,2]$. However, it is difficult to store and transmit stereo video due to the huge data amount. So, high efficient channel encoding algorithm and proper transmission strategy is needed to deal with the video transmission. In practical channel, especially in wireless channel with limited bandwidth, signal errors will inevitably appear at the receiver as a consequence of channel fading, multipath, noise and so on. Stereo video is highly compressed data stream, and is very sensitive to error, which will decrease the quality of reconstructed image at the receiver. Therefore, high efficient error correction technology and transmission control strategy is needed [3]. Recently, with the deep research in LDPC, more and more people focus on the video communication based on LDPC code $[4,5]$.

In this paper, we mainly focus on the stereo video communication on AWGN channel with limited bandwidth using UEP scheme based on LDPC encoding. According to the different contribution to image reconstruction at the receiver, the video stream is divided into different parts [6], then UEP is used to protect the different bits stream in different level. The structure is shown in Figure 1.

\section{Bits Stream Structure and Data Partition of Stereo Video}

Conceptually, the structure of H.264 encoder is divided into two layers: Video Coding Layer (VCL) and Network Abstraction Layer (NAL). VCL provides high performance function in video compression, including common definitions of video compression, block, macroblock, sub-graph layer and so on. NAL is responsible for network abstraction, which provides different adaptive capacity for different networks and transmitting package with proper mode. NAL works in two kinds of mode: Single Slice mode and Data Partition mode. When using Data Partition mode, H.264 puts all variable length codes with the same data type together in each frame [6].

Head information includes head information, macroblock type, frame type, predicted residual of motion vectors, frame flag etc. In H.264, this part is called A segmentation, which is the most important part. Intra-frame segmentation is called B segmentation. It loads the coding mode and the correlation coefficient in intra frame blocks. B segmentation works under the effect of A segmentation. Compared with information of inter-frame information segmentation, intra-frame information can prevent further drift, thus it is more effective than inter-frame segmentation. Inter-frame segmentation is called $\mathrm{C}$ segmentation. It only includes the coding mode and the correlation coefficient in 


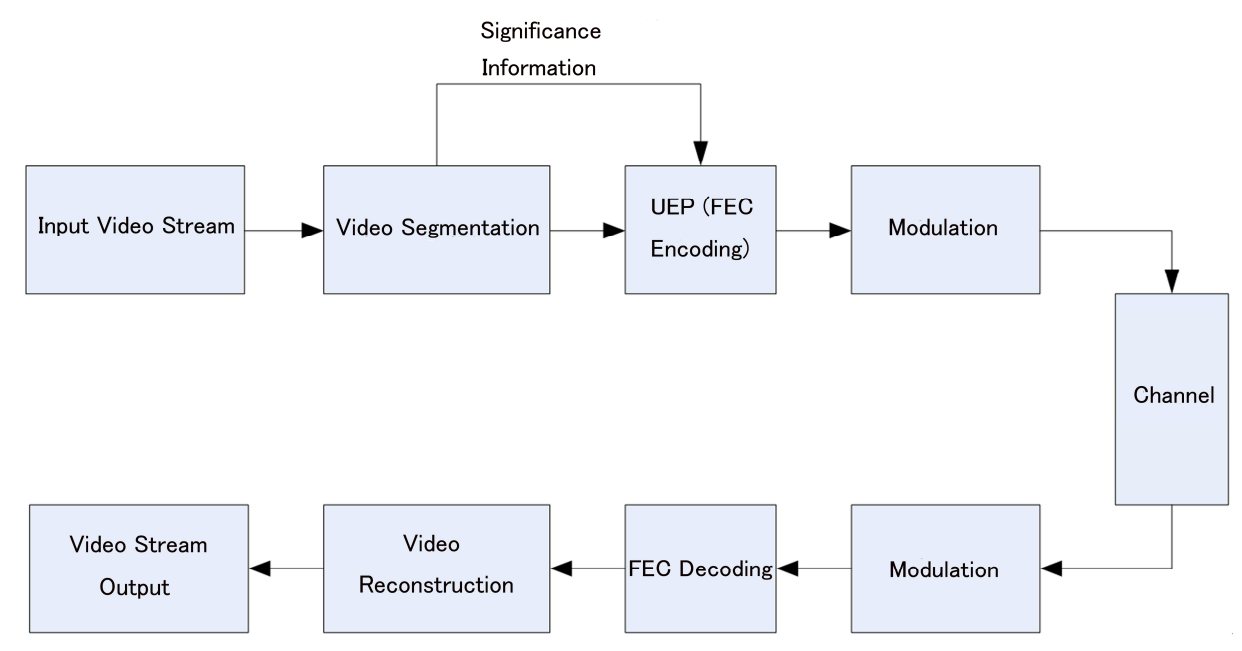

Figure 1. The diagram of stereo video communication based on UEP.

inter-frame blocks, it is the biggest segmentation in the video stream. Inter-frame segmentation is relatively subordinate, because it won't provide synchronous information in encoding or decoding.

Here, we use stereo video compression encoding based on H.264, the output bit stream has the same structure as that of H.264 encoder. Compared with the bit stream of H.264 encoder, the stereo video adds the disparity information of different video channel, such as disparity vector, disparity prediction model and predictive residual etc. Disparity vector and motion vector have the same important level. Once disparity vector goes wrong, the data of assisted video will be affected. So in this paper, we regard the slice head information, types of encoding frame, types of disparity prediction macroblock and disparity vector among different video channel as the same importance as the motion vector in one channel. Here, we divide the bit stream of stereo video into two parts: A segmentation and B segmentation .

A segmentation includes head information, MB-Type, Reference frame, motion vector prediction difference, disparity vector prediction difference, frame ending flag, the encoding mode and the correlation coefficient in intra frame, etc.

B segmentation includes the encoding mode and correlation coefficient in inter-frame.

We realize stereo video encoder based on H.264/AVC encoder platform. The parameters are listed in Table 1. The outputs of the encoder are two binary data

Table 1. The parameter of video encoder.

\begin{tabular}{|c|c|}
\hline Temporal direct conference frames & 5 \\
\hline Prediction mode & Modes of all blocks size \\
\hline Entropy coding method & CABAC \\
\hline $\begin{array}{l}\text { Motion estimation scope of the } \\
\text { search }\end{array}$ & \pm 32 \\
\hline $\begin{array}{l}\text { Disparity estimation scope of the } \\
\text { search }\end{array}$ & Level \pm 64 , Vertical: \pm 16 \\
\hline Structure of Group of picture & $\mathrm{IBBP} \ldots, \mathrm{N}=15, \mathrm{M}=3$ \\
\hline
\end{tabular}

file-test.264 and coding pursuit file-trace.txt [7,8]. We divide the bit stream according to these files.

The divided stereo video bit stream will be stored separately in file A and B. We adopt high-level protection to A bit stream because of its higher importance. For the $\mathrm{B}$ bit stream, we use low-level protection for the sake of encoding efficiency. At the receiver, if A segmentation data is lost, $B$ will be abandoned. If part of B segmentation is lost, the head information still can be used to improve the effect of error concealment $[9,10]$.

\section{Realization UEP Based on Different Rates Irregular LDPC Codes}

\subsection{The Principles of Realizing UEP Based on Different Rates Irregular LDPC Codes}

The key point of unequal error protection is: under the condition of limited bandwidth and the premise of prior protection to the important parts. We properly allocate the redundancy to the source and channel so as to reduce end-to-end distortion [11].

Assuming the total bit rate (the total bandwidth) is $R_{\text {total }}$, the parts of source and channel is $R_{S}$ and $R_{C}$ respectively. Suppose, the source is divided into $\mathrm{A}$ segmentation and B segmentation, so:

$$
R_{\text {total }}=R_{s}^{a}+R_{c}^{a}+R_{s}^{b}+R_{c}^{b}
$$

In formula (1): $R_{s}^{a}, \quad R_{s}^{b}$ represent the bits of A and B segmentation used in source encoding respectively. $R_{c}^{a}$, $R_{c}^{b}$ represent the bits of $\mathrm{A}$ and $\mathrm{B}$ bit stream used in the channel encoding respectively. Assuming the bit rate of channel encoding corresponding to A segmentation and B segmentation are $r_{a}, r_{b}$. Then, we can get the following formula:

$$
r_{a}=\frac{R_{s}^{a}}{R_{s}^{a}+R_{c}^{a}}, \quad r_{b}=\frac{R_{s}^{b}}{R_{s}^{b}+R_{c}^{b}}
$$




$$
D(R)=D_{S}\left(R_{S}^{a}, R_{S}^{b}\right)+D_{C}\left(R_{C}^{a}, R_{C}^{b}\right)
$$

Thus the question converts to: with the limited bandwidth and supposed data priority, the total distortion of the video at the receiver is minimized:

$$
\min (D(R))=\min \left(D_{s}\left(R_{s}^{a}, R_{s}^{b}\right)+D_{s}\left(R_{c}^{a}, R_{c}^{b}\right)\right)
$$

Subject to the following constraint conditions:

$$
\left\{\begin{array}{l}
R_{\text {total }}=R_{T} \\
r_{a} \leq r_{b}
\end{array}\right.
$$

where, $R_{T}$ represents the total channel bandwidth, and $D(R)$ represents the total distortion of source and channel, which is calculated by MSE. The relationship between PSNR and MSE is given by:

$$
P S N R=10 \lg \frac{255^{2}}{M S E}
$$

By adjusting the parameters of channel encoding and bit rate allocating between source encoding and channel reasonably, we can get the optimal reconstructed image at receiver.

UEP needs to allocate bit-rate between source and channel, under the condition that the total bandwidth is decided. According to adjusting the parameters of source encoding or the bit rate of channel encoding, the situation will be different.

\subsection{The Performance of UEP When the Source Rate is Decided}

When the bit rate of source encoding is pre-assigned, UEP can be realized by just adjusting the bit rate of channel .In this paper, we compared the properties of UEP scheme and equal error protection (EEP) scheme $\left(r_{a}<r_{b}\right.$ or $\left.r_{a}=r_{b}\right)$ in the same bandwidth. When the total bandwidth is decided, and the bit rate of source is pre-assigned (QP is unchangeable), that is, in the formula (1), $R_{s}^{a}+R_{s}^{b}$ and $R_{\text {total }}$ are determined. From $R_{\text {total }}=R_{s}^{a}+R_{c}^{a}+R_{s}^{b}+R_{c}^{b}$, we can know that the bits allocated to channel is also determined. UEP can be realized by adjusting the bit rate of channel encoding with different grade importance, that is by choosing $r_{a}, r_{b}$ to determine $R_{c}^{a}$ and $R_{c}^{b}$, so as to add different redundancy to information with different importance.

After analyzing the segmentations of stereo video bit stream, we found that the ratio of A segmentation and B segmentation is about 1:3 in Race 1 sequence. In order to keep the total bandwidth unchanged, the parameters of error correcting code are shown in the Table 2.

We use irregular LDPC code based on IeIRA permutation matrix with three different encoding rate in the experiment. They are $1 / 4,1 / 2$ and $3 / 4$, respectively. The code length of LDPC is 4064 bits, using BP decoding, the maximum iteration number is 80 . The length of Turbo code is 3568 bits. When using UEP scheme, the LDPC code with $1 / 4$ rate is used to protect the important data (the bit stream from A group), the LDPC code with 3/4 rate is used to protect the less important data(the bit stream from B group). If A segmentation is lost, B segmentation will be abandoned at the receiver.

In the experiment, we test the properties of UEP, EEP based on LDPC code and Turbo code in the AWGN channel with limited bandwidth by using BPSK modulation. Test sequences-Racel sequences from Japanese KDDI lab are used. The image size is $320 \times 240$.

At first, we give the BER performance of 3 kinds of code in AWGN channel in Figure 2. Clearly, not only the properties of irregular LDPC code based on IeIRA permutation matrix are better than Turbo code with the same length, but also error floors is lower than Turbo code. Figure 3 gives the differences of PSNR properties of image reconstructed by different protection scheme in the case of fixed source rate. (QP is 30 in stereo video compression encoding). Figure 4 shows the reconstructed 7 th frame main and assistant video by different encoding strategy at the receiver when SNR is $2.1 \mathrm{~dB}$.

Figure 3 and Figure 4 show that the UEP scheme based on LDPC code is always better than the other two schemes based on EEP. This is because we can reduce the error probability of important information by adding more redundancy in the important part of video stream. With the improvement of channel condition(increase of signal-to-noise ratio), this advantage is decreased $(\mathrm{Eb} / \mathrm{No}$ from 1.8 to 2.4, the advantage of main video stream based on LDPC UEP relative to LDPC EEP is reduced from 2.1 $\mathrm{dB}$ to $0.8 \mathrm{~dB}$ ). What's more, because of the excellent performance of LDPC code, the performance of EEP scheme based on LDPC code is better than that based on Turbo code.

Table 2. The parameters of various channel coding.

\begin{tabular}{|l|c|c|}
\hline \multirow{2}{*}{$\begin{array}{l}\text { Encoding } \\
\text { Scheme }\end{array}$} & \multicolumn{2}{|c|}{ Channel Encoding Rate } \\
\cline { 2 - 3 } & Segmentation $\boldsymbol{A}$ & Segmentation $\boldsymbol{B}$ \\
\hline LDPC UEP & $1 / 4$ & $3 / 4$ \\
\hline LDPC EEP & \multicolumn{2}{|c|}{$1 / 2$} \\
\hline Turbo EEP & \multicolumn{2}{|c|}{$1 / 2$} \\
\hline
\end{tabular}

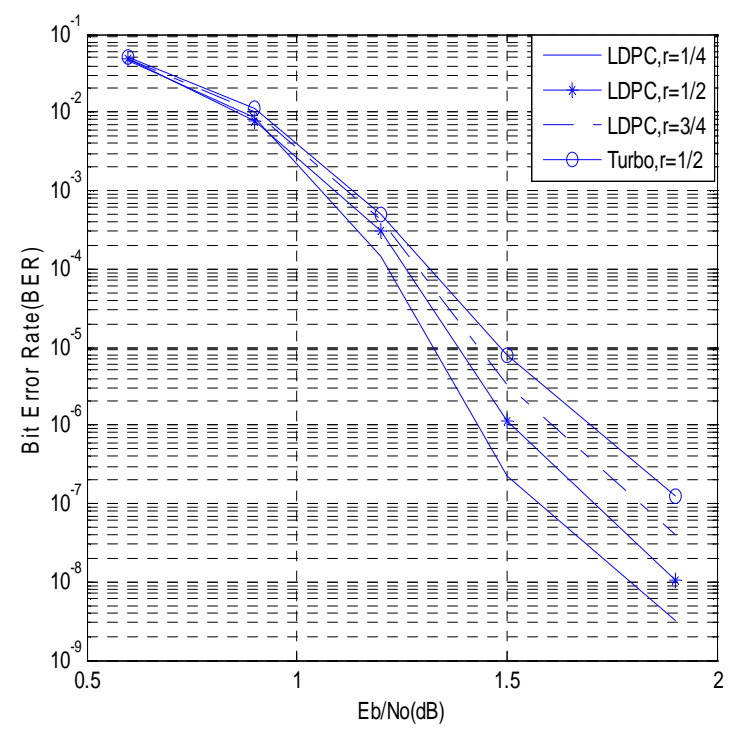

Figure 2. Performance of different channel code. 

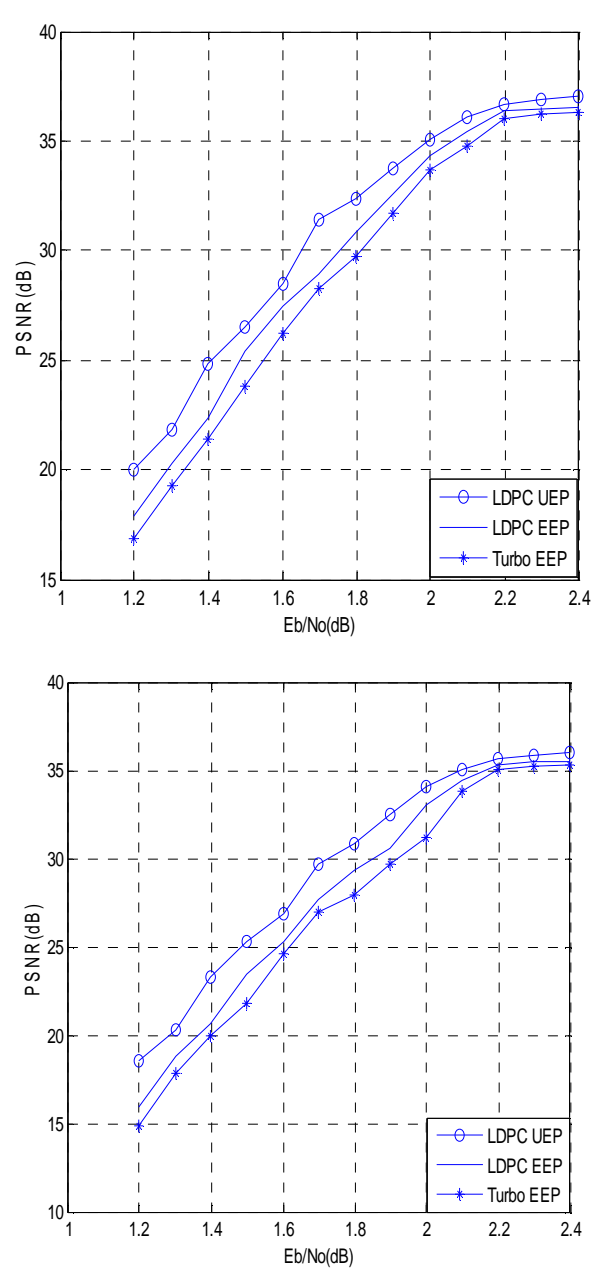

Figure 3. PSNR Compare of main, assistant video stream in different transmission scheme.

Figure 4 shows the reconstructed $7^{\text {th }}$ frame at the receiver. It is clear that Figure 4(a) is better than Figure 4(b) and Figure 4(c), and Figure 4(b) is a little bit better than Figure 4(c). On condition that $r_{a}<r_{b}$, it ensure the important data of A segmentation is fully protected, so we can get better subjective effect by using UEP scheme.

\subsection{The Performance of UEP in Case of Fixed Channel Encoding Rate}

When the total bandwidth is given, we also can pre-assigned the channel encoding rate, change the bit rate of the source (change QP) to adjust redundancy allocation between source and channel to get joint optimization .We will give the performance of different encoding strategies with fixed channel rate. The rate of LDPC code used in each encoding strategy is shown in Table 3.

The total bandwidth is fixed (for example $1.5 \mathrm{Mbps}$ ). When we use above mentioned three encoding strategies, the QP is 31, 26, and 28 in turn. The PSNR of reconstructed image by using different encoding strategies at the receiver are given as follows.
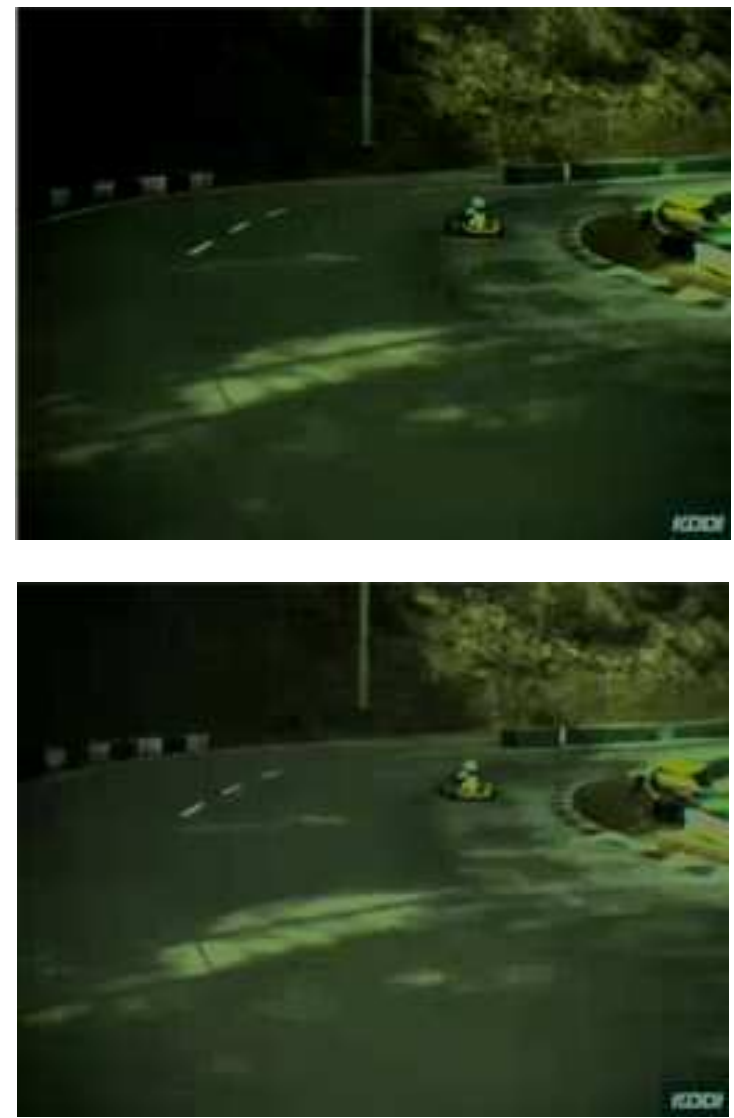

(a) LDPC UEP
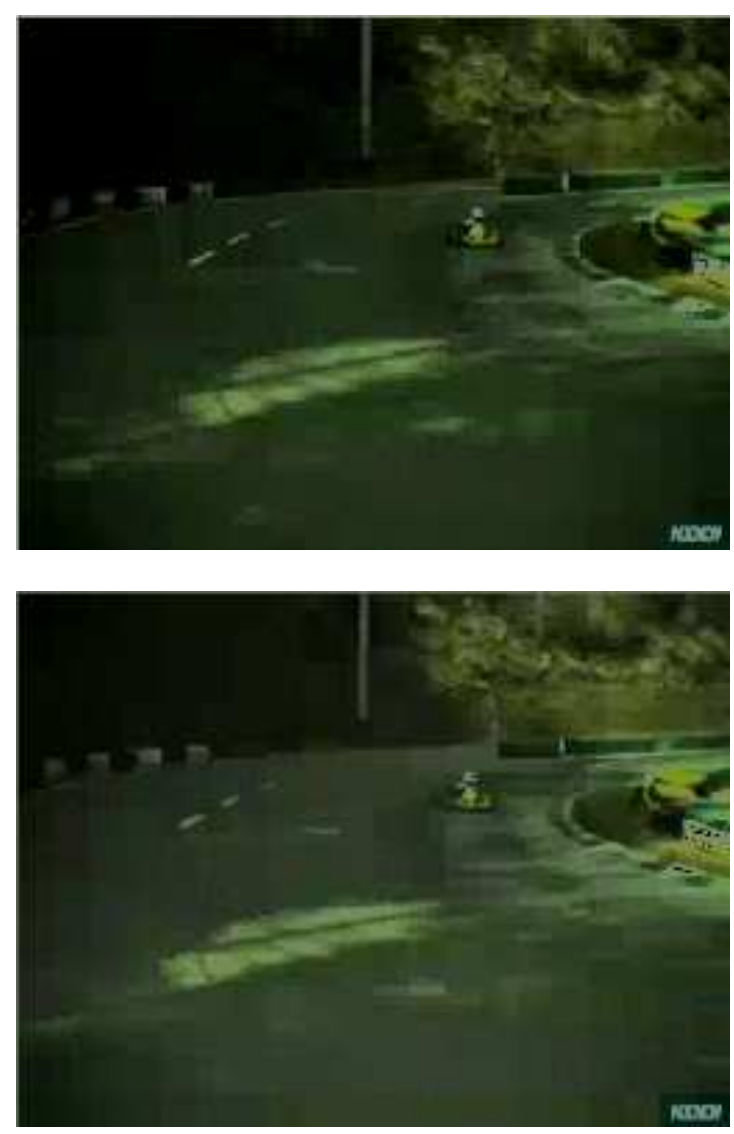

(b) LDPC EEP 

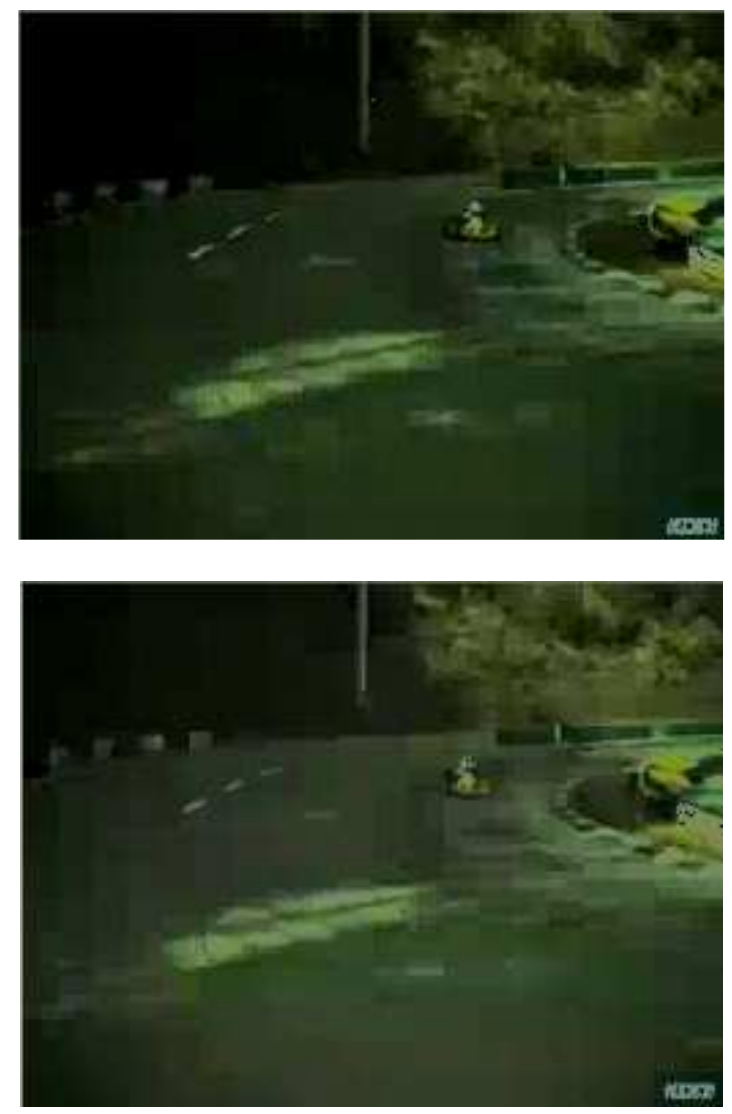

(c) Turbo EEP

Figure 4. The reconstructed main and assistant image of different transmission scheme at receiver.

Table 3. The channel coding RATE.

\begin{tabular}{|l|c|c|}
\hline \multirow{2}{*}{ Encoding Scheme } & \multicolumn{2}{|c|}{ Channel Encoding Rate } \\
\cline { 2 - 3 } & A Segmrntation & B Segmentation \\
\hline EEP1 & $1 / 4$ & $1 / 4$ \\
\hline EEP2 & $1 / 2$ & $1 / 2$ \\
\hline UEP & $1 / 4$ & $1 / 2$ \\
\hline
\end{tabular}

Figure 5 shows that EEP1 scheme can obtain the optimal performance for both main video and assistant video in low SNR condition. As the channel condition is going better, the performance of UEP gets over that of EEP1 at $1.8 \mathrm{~dB}$, EEP2 is exceeding the other two schemes when SNR is $2.1 \mathrm{~dB}$. The reason lies in that, in low SNR, channel error is the main factor affecting reconstruction quality in the receiver, so EEP1 scheme can get the best performance by using lower rate channel encoding strategy. When the channel is good, the bit error rate will reduce, and channel error will not be the main factor affecting PSNR of reconstructed image. Compared to EEP2, EEP1 and UEP have over protection, so they are worse than EEP2 in high SNR condition.

\section{Conclusions}

In this paper, we study the characteristics of stereo video bit stream based on H.264. and stereo video communication
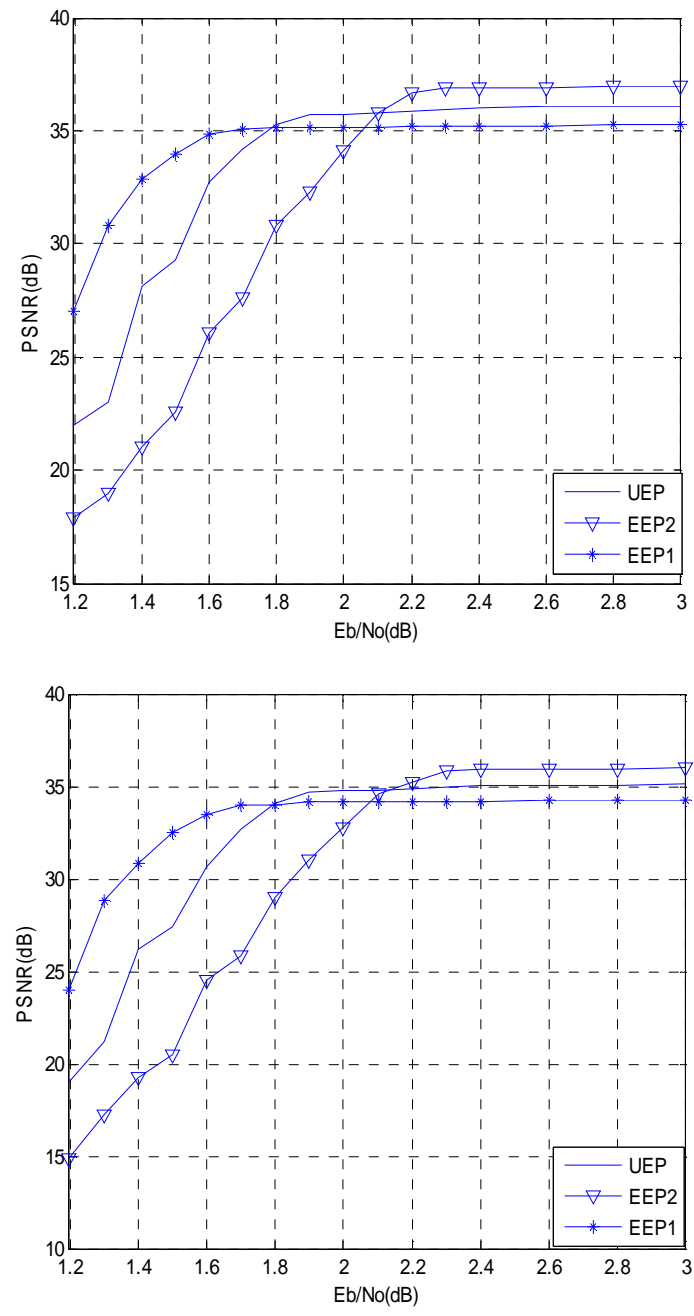

Figure 5. PSNR compare of main, assistant video stream in different transmission scheme (channel encoding is fixed, QP is changeable).

based on UEP scheme of LDPC code. According to the different importance to video reconstruction, we divide the stereo video into A and B segmentation. Then, according to the importance, UEP is used to get the best reconstructed image at the receiver.

According to source rate is fixed or channel rate is fixed when realizing UEP, we put forward two implementation algorithm. After analyzed the properties in the AWGN channel with fixed bandwidth, we obtain the following conclusions:

1) When the source rate is fixed ( $Q P$ is fixed), the performance of UEP scheme is always better than that of EEP. UEP can correct error with different abilities for different data, which gives more protection on more important data. Especially in the case of poor channel condition, the UEP scheme has advantage obviously.

2) When the channel code rate is fixed, we need to change the bit rate of source encoding to adapt to the requirement of bandwidth. After simulation, we find the scheme using low channel code rate have better performance than the scheme using high channel code 
rate in low SNR condition. As the channel condition is improving, channel error will not be the main factor affecting bit error, the scheme using low channel code rate is worse than that using high channel code rate on the contrary.

\section{References}

[1] J. Konrad, "Visual communication of tomorrow: natural," IEEE Communication Magazine, Vol. 39, No. 1, pp. 126-133, 2001.

[2] H. Kalva, L. Christodoulou, L. Mayron, O. Marques, and B. Furht, "Challenges and opportunities in video coding for 3D TV," ICME 2006, International conference on multimedia \& Expo.

[3] G. Gagnon, S. Subramaniam, and A. Vincent, "3-D MPEG-2 video transmission over broadband network and broadcast channels," Stereoscopic Displays and Virtual Reality Systems VIII, Proceedings SPIE, Vol. 4297, pp. 290-298, 2001.

[4] L. Wang, “Application of LDPC for digital TV terrestrial broadcasting system," Nanjing, China, A Dissertation Submitted to Southeast University for the Academic Degree of Master, March 2006.

[5] Q. Liu, Y. Lu, W. S. Wang, H. J. Cui, K. Tang, "Robust video transmission scheme using dynamic rate selection LDPC and RS codes," IMACS Multiconference on
Computational Engineering in Systems Applications (CESA), Beijing, China, October 4-6, 2006.

[6] T. Stockhammer, M. Hannuksela, and T. Wiegand, "H. 264/AVC in wireless environments," IEEE Transactions on Circuits and Systems for Video Technology, Vol. 13, pp. 657-673, July 2003.

[7] ITU-T Rec. H. 264/AVC /ISO/IEC 11496-10, “Advanced video coding," Final Committee Draft, Document JVT-G050, 2003.

[8] J. Ribas-Corbera, P. A. Chou, and S. Regunathan, "A generalized hypothetical reference decoder for H.264/ AVC," IEEE Transactions on Circuits and Systems for Video Technology, Vol. 13, pp. 674-687, July 2003.

[9] M. Yin and H. Y. Wang, "A scheme for H.264-based data partition and unequal error protection," Journal of Huazhong University of Science and Technology (Nature Science Edition), Vol. 34, July 2006.

[10] T. Wiegand, H. Schwarz, A. Joch, F. Kossentini, and G. J. Sullivan, "Rate-constrained coder control and comparison of video coding standards," IEEE Transactions on Circuits and Systems for Video Technology, Vol. 13, No. 7, pp. 688-703, July 2003.

[11] P. Y. Yip, J. A. Malcolm, W. A. C. Fernando, K. K. Loo, and H. K. Arachchi, "Joint source and channel coding for H.264 compliant stereoscopic video transmission," in Canadian Conference on Electrical and Computer Engineering, Saskatoon, Canada, May 2005. 